\title{
Effects of apparatus adaptation and ethanol upon runway performance'
}

\author{
KEITH A. WOLLEN, ALLEN R. DOBBS ${ }^{2}$ AND ROBERT L. SCHALOCK 3 \\ WASHINGTON STATE UNIVERSITY
}

Rats given ethanol ran faster on the first 2 days of ac quisition but slower on the last 2 days than placebo controls. However, when the $S s^{\prime}$ fear of the apparatus was extinguished by prior exploration of the alley, alcohol Ss tended to nu slower throughout training. It was concluded that alcohol improves performance in fear producing situations, but impairs performance when fear is reduced. Ss adapted to the apparatus ran faster throughout training than non-adapted Ss.

A consistent finding is that Ss given ethanol run faster on early acquisition trials than do placebo controls (Barry, Wagner, \& Miller, 1962; Nelson \& Wollen, 1965). Barry et al suggested that this may result from alcohol reducing the Ss' fear of the runway. If so, and if alcohol impairs performance in non-fearful situations, then the superiority of alcohol Ss should disappear if fear were extinguished by prior adaptation to the apparatus. This hypothesis was tested by comparing the performance of placebo and alcohol groups as a function of the presence or absence of free exploration of a runway.

A second goal was to examine the effects of alcohol and adaptation upon speeds at the end of training. If fear extinguishes during training, and if alcohol impairs performance in non-fearful situations, placebo Ss should run faster at the end of training than Ss given alcohol. Moreover, adapted Ss should mun faster than non-adapted Ss at the beginning of training, but no difference would be expected after sufficient training was given to extinguish the apparatus fear of nonadapted Ss.

Method

The Ss were 40 experimentally naive, male, hooded rats which ranged from 104 to 121 days of age on the first day of acquisition. Throughout the experiment, the Ss were maintained on a $23-\mathrm{hr}$. food and water deprivation schedule which permitted $1 \mathrm{hr}$. of ad lib consumption daily. The apparatus consisted of a 12-in. start box, a 36-in. runway, and a 12-in. goal box. The inside dimensions for each section were $3.5 \mathrm{in}$. wide by $4.7 \mathrm{in}$. high. The entire apparatus was painted flat black except for a transparent plastic lid. The start and goal boxes were each separated from the alley by a manually-operated, black, guillotine door. An additional motor-operated, transparent door was located adjacent to the black manual start door but on the runway side.

On the 7 th through 14th day of deprivation, each $S$ was handled for 2 to $3 \mathrm{~min}$. and received 10 Noyes $97 \mathrm{mg}$ reward pellets immediately prior to his datly feeding. On the 12th and 13th days of deprivation, Ss which were to be adapted to the apparatus were placed in the alley in groups of two or three and were allowed $1 \mathrm{hr}$. of free exploration each day with all doors raised and the clock starting and stopping as photobeams were intercepted. On the 14th day, each adapted $S$ was placed alone in the apparatus for $15 \mathrm{~min}$.

Training began on the 15 th day of deprivation and continued for 10 days. Each day began (about $1.5 \mathrm{hr}$. before the S's usual feeding time) with the administration of an alcohol (A) or a placebo, dextrose (D) solution. The A solution consisted of $15 \%$ ethanol in water, and the $\mathrm{D}$ solution was equated in caloric content to the A solution. In each case, the amount of solution administered was $1.5 \mathrm{cc}$ per $100 \mathrm{gm}$ of body weight. The solutions were heated to $100^{\circ} \mathrm{F}$ prior to administration via a stomach-loading procedure using Baxter $\mathrm{K}-31$ infant feeding tubes attached to a syringe.

The first trial of each day commenced $20 \mathrm{~min}$. after stomach loading. The black door was raised manually when S oriented toward it. The motor-operated door was raised automatically 2 sec. later. Interception of an infrared photobeam 6 in. down the alley started a clock which was stopped by the interception of a second beam 24 in. farther down the alley. Running times over this section were recorded to the nearest $.01 \mathrm{sec}$. and converted to speeds in $\mathrm{ft}$./sec. When the $\mathrm{S}$ entered the goal box, the goal box door was lowered. When $S$ finished eating one $97 \mathrm{mg}$ Noyes pellet, he was placed in a waiting cage for an intertrial interval of $75 \mathrm{sec}$. following which another trial was administered. Five such trials were given on the first day of acquisition, and 10 on each succeeding day.

The Ss were divided into four equal groups designated by the solution (A vs. D), and whether they had adaptation (a) or not (n). Thus the groups were Aa, $\mathrm{Da}, \mathrm{An}$, and Dn. In order to equate the daily alcohol intake of all animals, each $S$ given $D$ before running was given A immediately after running, and vice versa.

\section{Results and Discussion}

The mean running speeds are shown in Fig. 1 . The initial differences were examined by computing each S's mean speed over Days 1 and 2. Analysis of these scores revealed alcohol to be superior to dextrose for the non-adapted groups $(F=6.97, \mathrm{df}=1 / 18, \mathrm{p}<.025)$. In contrast, alcohol resulted in slower running when fear was reduced by adaptation. Although this latter difference did not reach statistical significance $(F=1.16)$, the data are in the predicted direction. The lack of a significant $\mathrm{Da}$ superiority over Aa might be 


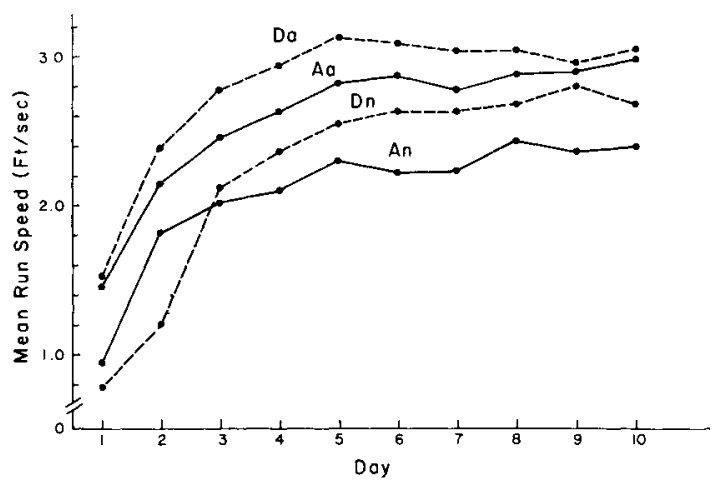

Fig. 1. Mean running speed as a function of days and condition.

attributed to residual fear from having received a stomach load for the first time. If present, such fear might depress the performance of Ss in Group Da. In contrast, fear or "anxiety" resulting from the loading procedure might have been reduced by alcohol in Group $\mathrm{Aa}$.

The tendency for $\mathrm{Da} \mathrm{Ss}$ to run faster than Aa Ss appears contrary to another study (Schalock \& Wollen, 1966) which used adaptation but found alcohol superior to dextrose initially. This apparent discrepancy may be due to differences in procedure. Schalock and Wollen administered a $30 \%$ solution of alcohol on the five days preceding the first day of acquisition, whereas the present study gave the same amount of a $15 \%$ solution for the first time on Day 1 of training. It is possible that a 15\% solution administered for the first time is more effective in reducing fear than the same amount of a $30 \%$ solution administered following five days of alcohol adaptation. It is also possible that dextrose $\mathrm{Ss}$ in the Schalock and Wollen study suffered from "hangover" effects (from the previous five days of alcohol) on the first day of acquisition. Hangovers in the alcohol group of their study might have been diminished by the alcohol administered to these Ss. Hangovers might depress the performance of the dextrose Ss.
An analysis of the mean speeds on the last two days of acquisition revealed a significant difference between Groups $\mathrm{Dn}$ and An $(F=9.94, \mathrm{df}=1 / 18, \mathrm{p}<.01)$. This finding is consistent with that of Nelson \& Wollen (1965), and suggests that alcohol impairs performance after sufficient training is given to extinguish the animal's fear. The expectation that the $\mathrm{Da}$ curve would end acquisition above the Aa curve was confirmed, but the difference was not significant on the last two days $(F<1.00)$.

As may be seen in Fig. 1, the expectation that adapted and non-adapted Ss would end acquisition at the same level was not confirmed. Group Dn shows a slight tendency to approach Group $\mathrm{Da}$, but Group An is well below Group Aa. Despite a general tendency for alcohol to inhibit performance on all but the initial trials, it is interesting that the Aa curve is above that for Dn at all points. This suggests that this group's facilitation resulting from adaptation was greater than their impairment produced by alcohol. The finding that the An curve was lower on Day 1 than the curves for adapted Ss suggests that, in the amounts given, adaptation is either more effective than alcohol in reducing fear, or that the adapted Ss benefited from "Iatent learning."

\section{References}

Barry, H., III, Wagner, A. R., \& Miller, N. E. Effects of alcohol and amobarbital on performance inhibited by experimental extinction. J. comp. physiol. Psychol., 1962, 55, 464-468.

Nelson, P. B., \& Wollen, K. A. Effects of ethanol and partial reinforcement upon runway acquisition. Psychon. Sci., 1965, 3, 135- 136.

Schalock, R. L., \& Wollen, K. A. The immediate and long-term effect on runway performance of reversing ethanol and dextrose conditions. Psychon. Sci., 1966, 4, 113-114.

\section{Notes}

1. This investigation was supported in part by funds provided for biological and medical research by the State of Washington Initiative Measure No. 171.

2. Now at the University of Iowa, Iowa City, Iowa.

3. Now at the Psychophysiology Laboratory, V. A. Hospital, American Lake, Washington. 